\title{
Characteristics of YBCO SQUIDs with Step-edge And Biepitaxial Grain Boundaries
}

\author{
S.Y. Yang, C.H. Chen, and H.E. Horng, \\ Department of Physics, National Taiwan Normal University, Taipei 117, Taiwan \\ W.L. Lee, and H.C. Yang \\ Department of Physics, National Taiwan University, Taipei 106, Taiwan
}

\begin{abstract}
Some characteristics of YBCO SQUIDs with stepedge and biepitaxial grain boundaries were investigated. V-I curves of the step-edge SQUIDs can be described by the RSJlike model with a linear relationship between the critical current $I_{c}$ and temperature $T$, whereas the typical $I_{c}-T$ curve for the biepitaxial SQUIDs concaved upward at temperatures near the critical temperature. The V-I curves of both kinds of the stepedge and the biepitaxial SQUIDs under microwave irradiation revealed integral Shapiro steps. Furthermore, the voltages of the step-edge SQUIDs were well modulated by the applied magnetic field. And also, the typical Fraunhofer diffraction of the $I_{c}-\Phi$ curve was observed for the single junction in the step-edge SQUIDs. On the other hand, the magnetic field modulated voltage oscillation was also found for the biepitaxial SQUIDs. All the details will be discussed in this report.
\end{abstract}

\section{I.INTRODUCTION}

Due to the development of superconducting integrated circuits, the fabrication and characterization of Josephson junctions and SQUIDs have become significant and important. At present, many groups have made dc $\mathrm{YBa}_{2} \mathrm{Cu}_{3} \mathrm{O}_{7-\mathrm{y}}(\mathrm{YBCO}) \mathrm{SQUIDs}$ by using different techniques, such as bicrystal substrate junctions [1], biepitaxial junctions [2], superconductor-normal metal-superconductor junctions [3], ramp-type junctions [4] and step-edge junctions [5]. However, the characteristics of SQUIDs with different grain boundaries were usually discussed individually. Thus, the similarities or the differences among these SQUIDs can hardly to be clarified. In this work, we investigate simultaneously the characteristics of the YBCO SQUIDs with step-edge and biepitaxial grain boundaries. The geometries of the step-edge and biepitaxial SQUIDs are shown in Fig. 1. The step angle used here is $50^{\circ}$. As a result, the most possible interface of the YBCO thin film at the grain boundary on the the $50^{\circ}$-step-edge $\mathrm{MgO}(001)$ substrate is $<203>$ [6]. However, the grain boundary is formed as $<100>\mid<110>$ for the biepitaxial YBCO thin film. It is clear that the grain boundaries of the step-edge and biepitaxial YBCO SQUIDs differ from each other. Thus, the properties of these two SQUIDs may exhibit different behaviors. To check this point, the characteristics of these two kinds of YBCO SQUIDs are examined and then compared with each other.

Manuscript received 15 September, 1998.

This work is supported by the National Science Council of ROC under grand Nos. NSC87-2112-M-003-008, NSC87-2112-M-003-001 and NSC87-2112-

\section{EXPERIMENTAL DETAILS}

An rf magnetron sputtering system was used to deposit all films on the $\mathrm{MgO}(001)$ substrates in this work. In order to obtain a sharp step-edge on the $\mathrm{MgO}$, the substrate was bombarded by Ar ion with the photoresist of AZ1518. On the other hand, to create a $45^{\circ}$ grain boundary for the biepitaxial YBCO SQUIDs, $\mathrm{CeO}_{2}$ was chosen to be a seed layer for the YBCO. This seed layer was achieved by using a lift-off method instead of a traditional ion milling process. The details have been discussed in our previous work $[2,7]$. Then, $2000 \AA$-thick YBCO films were sputtered onto the step-edge and biepitaxial substrates followed by patterning these two films with the aid of dilute hydrochloric acid. The hole area of each SQUID was $20 \times 40 \mu \mathrm{m}^{2}$, and the width of each junction is $4 \mu \mathrm{m}$. Thus, the self-inductance of the fabricated SQUIDs is estimated to be $44.4 \mathrm{pH}$ [8]. Finally, gold was evaporated onto the contact pads and copper wires were connected with indium to the gold contacts for electrical measurements.

To investigate the voltage-current curves of the YBCO SQUIDs under microwave irradiation, the SQUIDs were subjected to an ac electromagnetic field which was guided to

(a)

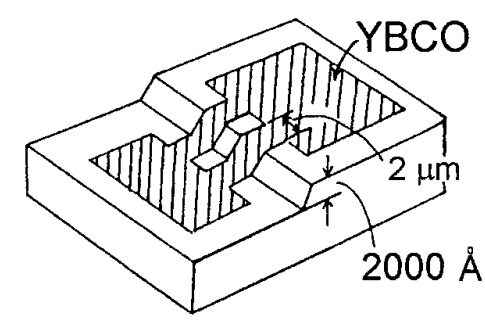

(b)

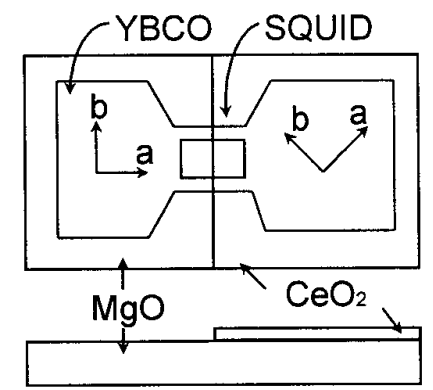

Fig. 1. Geometry of the (a) step-edge and (b) biepitaxial YBCO SQUIDs. The dimensions are not to scale. 


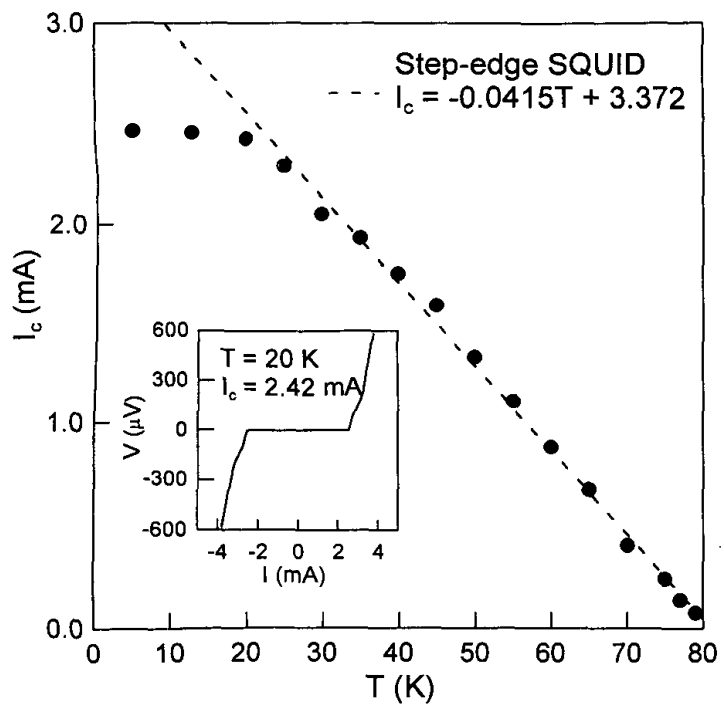

Fig. 2. The temperature dependent critical current of the step-edge YBCO SQUID. The V-I curve of the SQUID at $20 \mathrm{~K}$ is shown in the inset.

the sample through a dipole antenna. A solenoid was used to provide a magnetic field perpendicular to the hole of each SQUID. Moreover, the sample space was shielded from outside noises by mu-metal. The other experimental details have been illustrated elsewhere [2].

\section{RESULTS AND DISCUSSION}

A typical voltage-current curve of the step-edge YBCO SQUIDs is shown in the inset of Fig. 2. This curve exhibits the RSJ-like behavior with the critical current $I_{c}$ and the normal resistance $R_{n}$ being $2.42 \mathrm{~mA}$ and $0.7 \Omega$ at $20 \mathrm{~K}$, respectively. This gives to the product of $I_{c} R_{n}$ as $169.4 \mu \mathrm{V}$ at

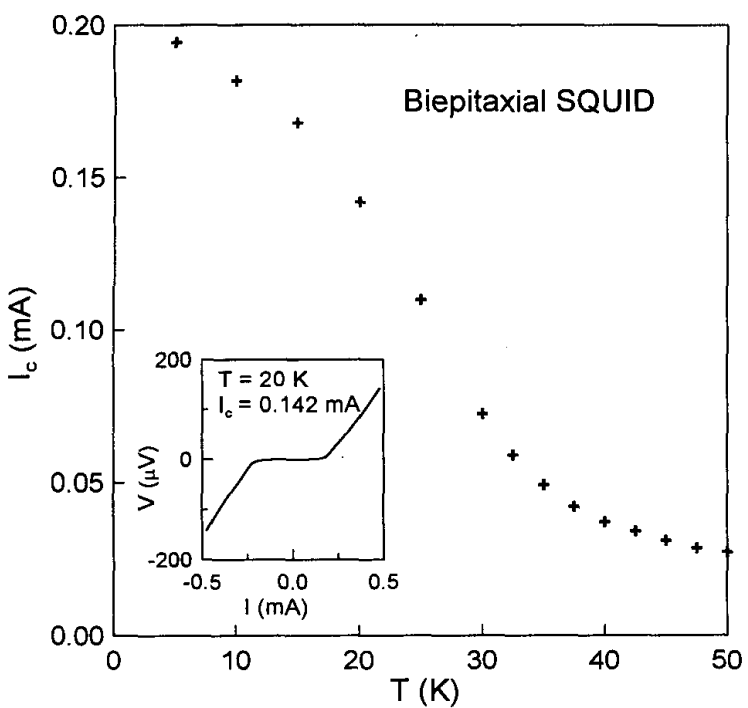

Fig. 3. Critical current as a function of temperature for the biepitaxial SQUID. The inset represents the V-I curve at $20 \mathrm{~K}$ for the biepitaxial SQUID.
$20 \mathrm{~K}$ for the step-edge SQUID shown here. By checking carefully the V-I curve of the step-edge SQUID, two knees can be found at $\mathrm{I}= \pm 2.42 \mathrm{~mA}$ and $\pm 3.15 \mathrm{~mA}$ respectively. This suggests that each step-edge Josephson junction consists of two junctions in series. The temperature dependent critical current of the step-edge SQUID is presented in Fig. 2. The critical current $I_{c}$ almost remains constant at temperature $T$ lower than $20 \mathrm{~K}$. With increasing $\mathrm{T}, \mathrm{I}_{\mathrm{c}}$ is reduced linearly down to zero at $\mathrm{T}=81 \mathrm{~K}$. This linearity reveals that the weak link of the step-edge YBCO junctions is similar to a superconductor-insulator-superconductor junction behavior [9]. This linear relationship between $I_{c}$ and $T$ was also found for our other step-edge SQUIDs.

For the YBCO biepitaxial SQUIDs, a typical V-I curve of these SQUIDs at $20 \mathrm{~K}$ is shown in the inset of Fig. 3. According to the V-I curve, the value of the $I_{c} R_{n}$ at $20 \mathrm{~K}$ was found to be $56 \mu \mathrm{V}$ for the biepitaxial YBCO SQUID. By our experimental results of the $I_{c} R_{n}$ for numerous step-edge and biepitaxial SQUIDs, a fact was observed that the $I_{c} R_{n}$ of the biepitaxial SQUIDs is smaller than that of the step-edge

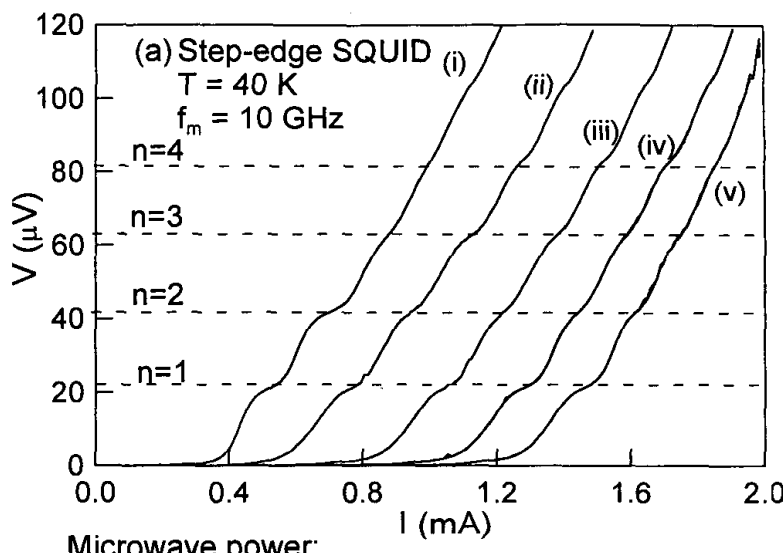
(i) $\circ \mathrm{dBm}$
(v) $-8 \mathrm{dBm}$
(ii) $-2 \mathrm{dBm}$
(iii) $-4 \mathrm{dBm}$
(iv) $-6 \mathrm{dBm}$

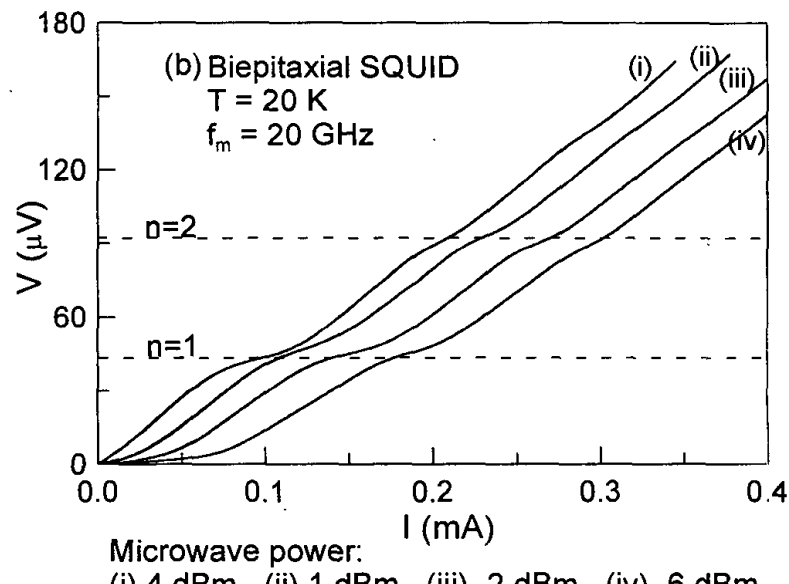

$\begin{array}{llll}\text { (i) } 4 \mathrm{dBm} & \text { (ii) } 1 \mathrm{dBm} & \text { (iii) }-2 \mathrm{dBm} & \text { (iv) }-6 \mathrm{dBm}\end{array}$

Fig. 4. The V-I curves inder microwave irradiation for (a) the step-edge SQUID and (b) the biepitaxial SQUID. 


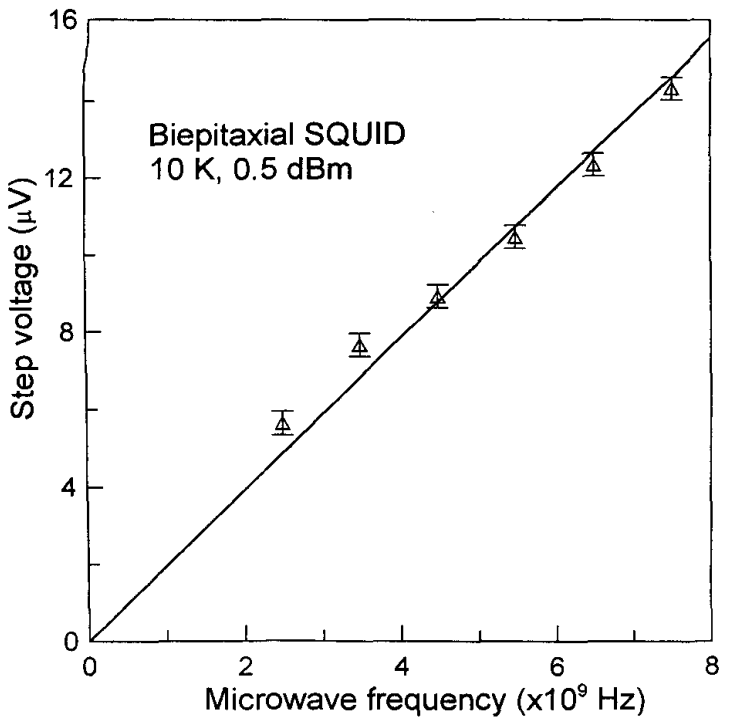

Fig. 5. The first-order step voltage versus the microwave frequency for the biepitaxial SQUID at $10 \mathrm{~K}$

SQUIDs at the same temperature. The reduction in the $I_{c} R_{n}$ of the biepitaxial SQUIDs with respect to the step-edge SQUIDs may have resulted from the considerably high-angle $\left(45^{\circ}\right)$ grain boundary in the a-b plane. The difference in the $I_{c} R_{n}$ 's between the two kinds of grain-boundary SQUIDs implies that different self-inductances are needed to optimize the SQUID devices. Thus, different SQUID patterns are required for different grain-boundary SQUIDs.

The critical currents of the biepitaxial SQUIDs at various temperatures were also measured and the typical results are shown in Fig. 3. Instead of a linear relationship between ' $I_{c}$ and $T$ for the step-edge SQUIDs, the $I_{c}-T$ curve concaves upward at temperatures higher than $20 \mathrm{~K}$. It is well known that the temperature dependent critical current is dominated by the Josephson coupling of the Josephson junctions of the SQUID. Thus, the experimental results reveal that the interface orientation of the grain boundary did affect the Josephson coupling behavior for the junctions of the SQUIDs.

In Fig. 4 , the voltage-current curves of both the step-edge and the biepitaxial SQUIDs under microwave irradiation are shown. The temperature $T$, microwave frequency $f_{m}$ and power are labeled in the Figs. 4(a) and 4(b) for the step-edge SQUID and the biepitaxial SQUID respectively. Both of the two kinds of SQUIDs exhibit integral Shapiro steps with the corresponding first order constant-voltage steps of $20.7 \mu \mathrm{V}$ and $41.4 \mu \mathrm{V}$. Through a careful inspection, the amplitude of a given order step was found to be modulated by the microwave power slightly. When varying the microwave frequency $f_{m}$, the rf-induced constant-voltage steps in the V-I curves were observed to be shifted proportionally to $f_{m}$. A typical result for the first order constant-voltage step of the biepitaxial SQUID was shown in Fig. 5. According to the experimental data, the slope of the $\mathrm{V}-\mathrm{f}_{\mathrm{m}}$ curve was obtained as $\mathrm{V} / \mathrm{f}_{\mathrm{m}}=2.0 \times 10^{-15}$ Webber. This is in good agreement

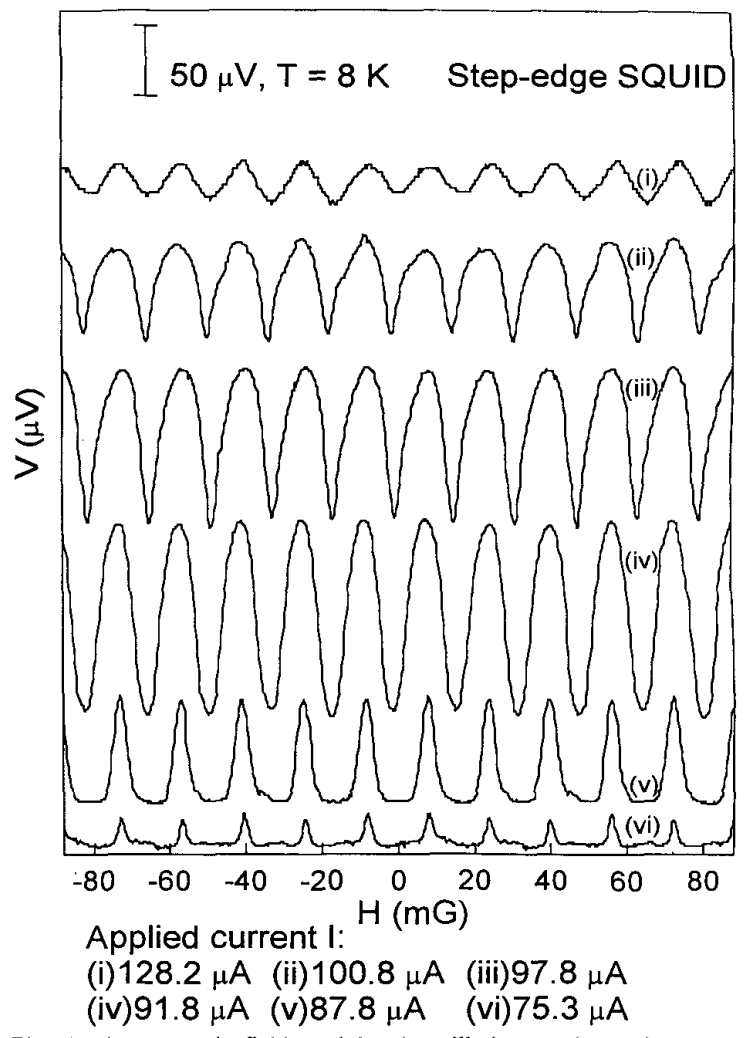

Fig. 6. The magnetic field modulated oscillations under various applied current for the step-edge SQUID at $8 \mathrm{~K}$.

with the theoretically expected result of $h / 2 \mathrm{e}$ for the firstorder rf-induced constant voltage step [10].

The magnetic field modulated voltages of one of the fabricated step-edge YBCO SQUIDs at $8 \mathrm{~K}$ are presented in Fig. 6. The applied current $\mathrm{I}$ is varied from $75.3 \mu \mathrm{A}$ to $128.2 \mu \mathrm{A}$. It was found that the peak-to-peak voltage $V_{p p}$ becomes larger as $I$ is increased and reaches a maximum of $130 \mu \mathrm{V}$ under $\mathrm{I}=91.8 \mu \mathrm{A}$. By increasing $\mathrm{I}$ higher than 91.8 $\mu A$, the $V_{p p}$ starts to decrease. The period of the applied magnetic field in Fig. 6 is observed to be $11.85 \mathrm{mG}$. This implies that the effective hole area of the step-edge SQUID is $1730 \mu \mathrm{m}^{2}$. Since the geometric hole area of the SQUID is 800 $\mu \mathrm{m}^{2}$, the focusing factor of the step-edge YBCO SQUID is around 2.16. By enlarging the range of the applied magnetic field, the magnetic field modulated voltage $(\mathrm{V}-\Phi)$ and the critical current $\left(\mathrm{I}_{\mathrm{c}}-\Phi\right)$ relationships of the junctions in the SQUID are examined. These two curves for the single junction of the step-edge SQUID at $20 \mathrm{~K}$ are shown in Figs. 7 (a) and 7(b). It was observed that V- $\Phi$ curve oscillates out of phase with respect to the $I_{c}-\Phi$ curve. In addition, the experimental $I_{c}(\Phi)$ data were found to follow the Fraunhafer diffraction pattern with some deviations, as shown in Fig. 7(b) in which the solid line denotes the Fraunhofer diffraction pattern. These deviations are believed to be due to the inhomogeneities in the critical current distribution along the grain boundary. 


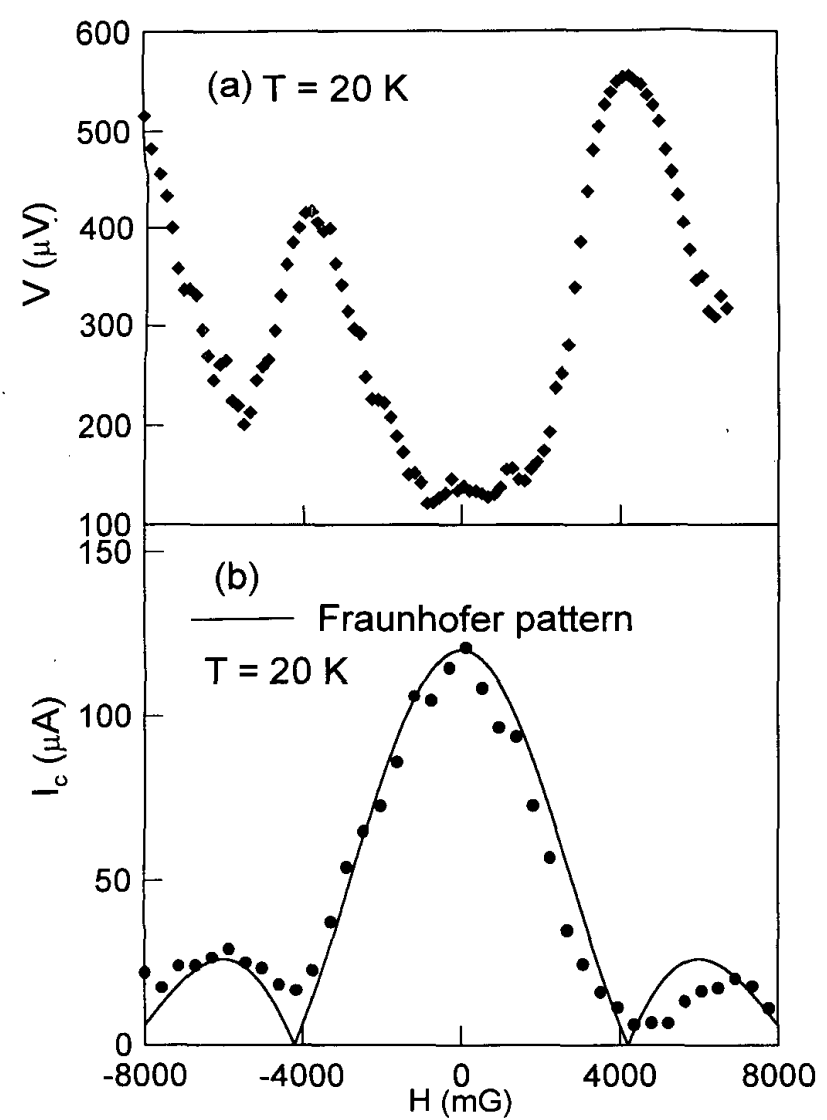

Fig. 7. Magnetic field modulated (a) voltage and (b) critical oscillations for the junction of the step-edge YBCO SQUID.

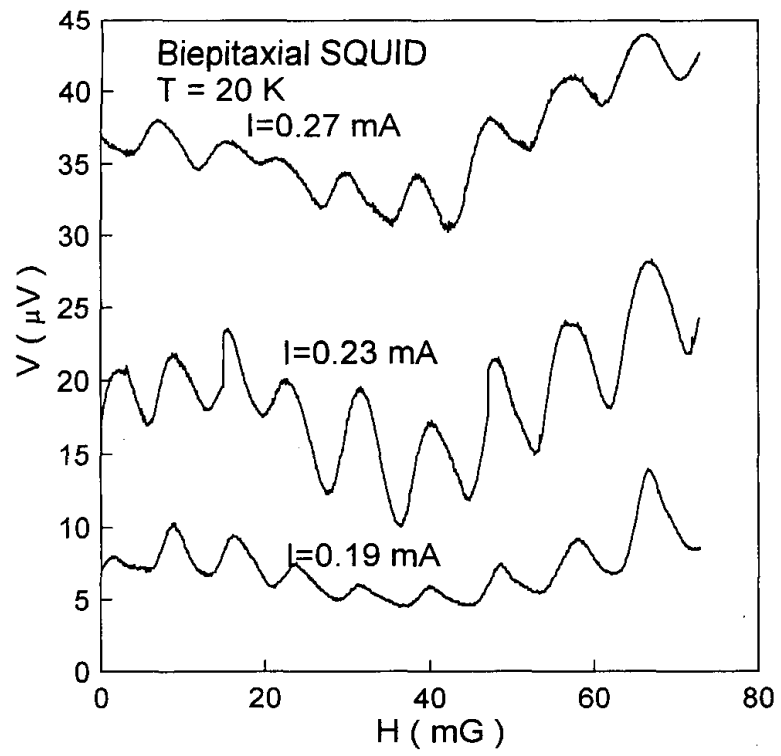

Fig. 8. The voltage of the biepitaxial SQUID is modulated by the applied magnetic field with various applied current at $20 \mathrm{~K}$.
The V- $\Phi$ curves of the biepitaxial YBCO SQUIDs with various applied currents I were also examined. A typical result at $20 \mathrm{~K}$ is shown in Fig. 8. The maximum peak-to. peak voltage is about $10 \mu \mathrm{V}$ and the period of the applied magnetic field is found to be $8.64 \mathrm{mG}$. As a result, the corresponding effective hole area of the SQUID is $2460 \mu \mathrm{m}^{2}$. Since the hole area of the biepitaxial SQUID is $800 \mu^{2}$ ? which is the same as that of the step-edge SQUID, the focusing factor of the YBCO biepitaxial SQUID is about 2.99. This value is close to that of the above step-edge YBCO SQUID. This coincidence between the focusing factors of the two kinds of the SQUIDs suggests that the focusing factor is almost independent of the orientation of the grain boundary but mainly dominated by the SQUID geometry.

\section{CONCLUSIONS}

By comparing the experimental results for the step-edge and biepitaxial YBCO SQUIDs, we found that the orientation of the grain boundary affected the Josephson coupling behavior greatly. Integral constant-voltage steps in the V-I curves were observed for both the step-edge and biepitaxial SQUIDs under microwave irradiation. And also, the observed value of $\mathrm{h} / 2 \mathrm{e}$ is in good agreement with the theoretically expected result. The voltages of both the step-edge and biepitaxial YBCO SQUIDs are well modulated by the applied magnetic field. Furthermore, the focusing factors of a SQUID is mainly dominated by its geometric pattern and almost independent of the orientation of the grain boundary.

\section{REFERENCES}

[1]D. Dimos, P. Chaudhari, and J. Mannhart, "Superconducting transport properties of grain boundaries in $\mathrm{YBa}_{2} \mathrm{Cu}_{3} \mathrm{O}_{7}$ bicrystals", Phys. Rev. $\mathrm{B}$ 41, 4038-4049(1990)

[2]H.E. Horng, S.Y. Yang, W.L. Lee, H.C. Yang, and J.M. Wu, "Biepitaxial grain boundary $\mathrm{YBa}_{2} \mathrm{Cu}_{3} \mathrm{O}_{7-y}$ thin-film SQUIDs", pp.683-686, Applied Superconductivity 1997: Proceedings of EUCAS 1997(Institute of Physics Publishing, Bristol and Philadelphia)

[3]K.A. Delin and A.W. Kleinsasser, "Stationary properties of high critical temperature proximity effect Josephson junctions", Supercond. Sci. Technol., 9, 227-269(1996)

[4]C. Hortmann, P. Leinenbach, R. Dittmann, U. Hartmann, and A.I. Braginski, "Correlation between ramp morphology and peoperties of ramp-type junctions", IEEE Trans. Appl. Supercond., 7, 28442847(1997)

[5]L.C. Ku, H.M. Cho, J.H. Lu, S.Y. Wang, W.B. Jian, H.C. Yang, and H.E. Horng, "Characteristics of $\mathrm{YBa}_{2} \mathrm{Cu}_{3} \mathrm{O}_{\mathrm{y}}$ step-edge Josephson junctions on MgO sunstrate", Physica C, 229, 320-324(1994)

[6]C.P. Foley, S. Lam, B. Sankrithyan, Y. Wilson, J.C. Macfarlane, and L. Hao, "The effects of the step angle on step edge Josephson junctions on MgO", IEEE Trans. Appl. Supercond., 7, 3185-3188(1997)

[7]S.Y. Yang, H.E. Horng, W.L. Lee, H.W. Yu, and H.C. Yang, "Fabrication of biepitaxial $\mathrm{YBa}_{2} \mathrm{Cu}_{3} \mathrm{O}_{7-\mathrm{y}}$ Josephson junctions and SQUIDs", Chin. J. Phys., 36, 409-415(1998)

[8]M.B. Ketchen, W.J. Gallagher, A.W. Kleinsasser, S. Murphy, and John R. Clem, "DC SQUID focuser", pp.865-871, SQUID'85-Superconducting Quantum Interference Devices and their Applications(Walter de Gruyter \& Co., 1985)

[9]V. Amegaokar and A. Baratoff, "Tunneling between superconductor:" Phys. Rev. Lett., 10, 486-489(1963), Phys. Rev. Lett., 10, 489(1963)

[10]S. Shapiro, "Josephson current in superconducting tuneling: The effect of microwaves and other observations", 11, 80-82(1963) 